\title{
INVect - a novel polycationic reagent for transient transfection of mammalian cells
}

\author{
Sebastian Püngel ${ }^{1}$, Miklos Veiczi ${ }^{2}$, Tim Welsink1, Daniel Faust ${ }^{1}$, Vanessa Vater ${ }^{1}$, Derek Levison², Uwe Möller \\ Wolfgang Weglöhner ${ }^{1 *}$ \\ From 23rd European Society for Animal Cell Technology (ESACT) Meeting: Better Cells for Better Health \\ Lille, France. 23-26 June 2013
}

\section{Background}

For rapid recombinant protein production in small to medium size volumes, transient transfection of mammalian cells is still the method of choice in biotechnology [1]. However, due to the high costs of commercially available lipofectamines or polycationic transfection reagents such as polyethylenimine (PEI), the most widely used transfection reagents available present a substantial economic bottleneck. While these reagents produce seemingly high transient transfection rates [2], there is still a strong desire for transfection reagents providing both secure and easy handling and higher recombinant protein production. As part of our commitment to excellence, InVivo BioTech Services initiated a joint venture with emp Biotech and developed a novel polycationic reagent, named INVect, for transient transfection and recombinant protein production in mammalian cells.

\section{Materials and methods}

Mammalian cells were cultured in CD-ACF media using shake flasks and standard culture conditions. Cells were transfected with $10 \mu \mathrm{g}$ per $\mathrm{mL}$ of a GOI harboring plasmid at a cell density of $5 \times 10^{6}$ cells per $\mathrm{mL}$ in FreeStyle $^{\mathrm{TM}}$ Medium (Life Technologies) with INVect to DNA ratio of $6: 1(\mathrm{w} / \mathrm{w})$ and PEI to DNA ration of 2:1 $(\mathrm{w} / \mathrm{w})$. Cultures were supplemented with same volume Protein Expression Medium (Life Technologies) 2 hours post transfection. GFP and SEAP expression took place in $8 \mathrm{~mL}$ culture volume in $50 \mathrm{~mL}$ bioreactor tubes. Expression of other reporter proteins were performed in $150 \mathrm{~mL}$ culture volume in $500 \mathrm{~mL}$ shake flasks. Transfection efficiency was determined 24 hours post transfection by counting green fluorescent positive cells using

\footnotetext{
* Correspondence: wegloehner@invivo.de

'InVivo BioTech Services GmbH, 16761 Hennigsdorf, Germany

Full list of author information is available at the end of the article
}

a FACSCalibur (Becton, Dickinson and Company). SEAP expression was determined in cell culture supernatant on day 6 post transfection by a photometric pNPP turn-over assay. Quantification of IgG was performed by protein $G$ affinity chromatography on day 6 post transfection. Thrombomodulin concentration was calculated from cell culture supernatant on day 6 post transfection by IMUBIND ${ }^{\circledR}$ Thrombomodulin ELISA Kit (american diagnostica). His-tagged recombinant protein was purified on day 6 post transfection by TALON ${ }^{\circledR}$ immobilized metal affinity chromatography system.

\section{Results}

Cytotoxicity was tested over a broad range of concentrations. Results demonstrate several novel synthetic polymers exhibiting transfection efficiencies even higher than common PEIs after optimized ratios of DNA-topolymer were applied. Transfection efficiency of INVect was compared to PEI, currently the standard transfection reagent for transient gene expression. INVect was found to generally give better transfection efficiencies of greater $80 \%$ in a GFP assay (Figure 1A). Batch-to-batch reproducibility was shown on five independent INVect batches. Transfection results were highly consistent and in the range of $80-90 \%$ (Figure 1B).

INVect successfully delivers genes to HEK293-F, CHO-S and CAP-T cells as shown in a SEAP expression system (Figure 1C). Post-transfection cell productivity was determined under TGE manufacturing conditions. Thrombomodulin $(60 \mathrm{kDa})$ (Figure 1D), an IgG (144 $\mathrm{kDa}$ ) (Figure 1E) and a HIS-tagged Protein of Interest $(\sim 40 \mathrm{kDa})$ (Figure 1F) were transiently expressed using INVect as transfection reagent and conventional $25 \mathrm{kDa}$ PEI as control. Cells were transfected with a gene of interest harboring plasmid, with product concentration being measured on day 6 post transfection. The use of 


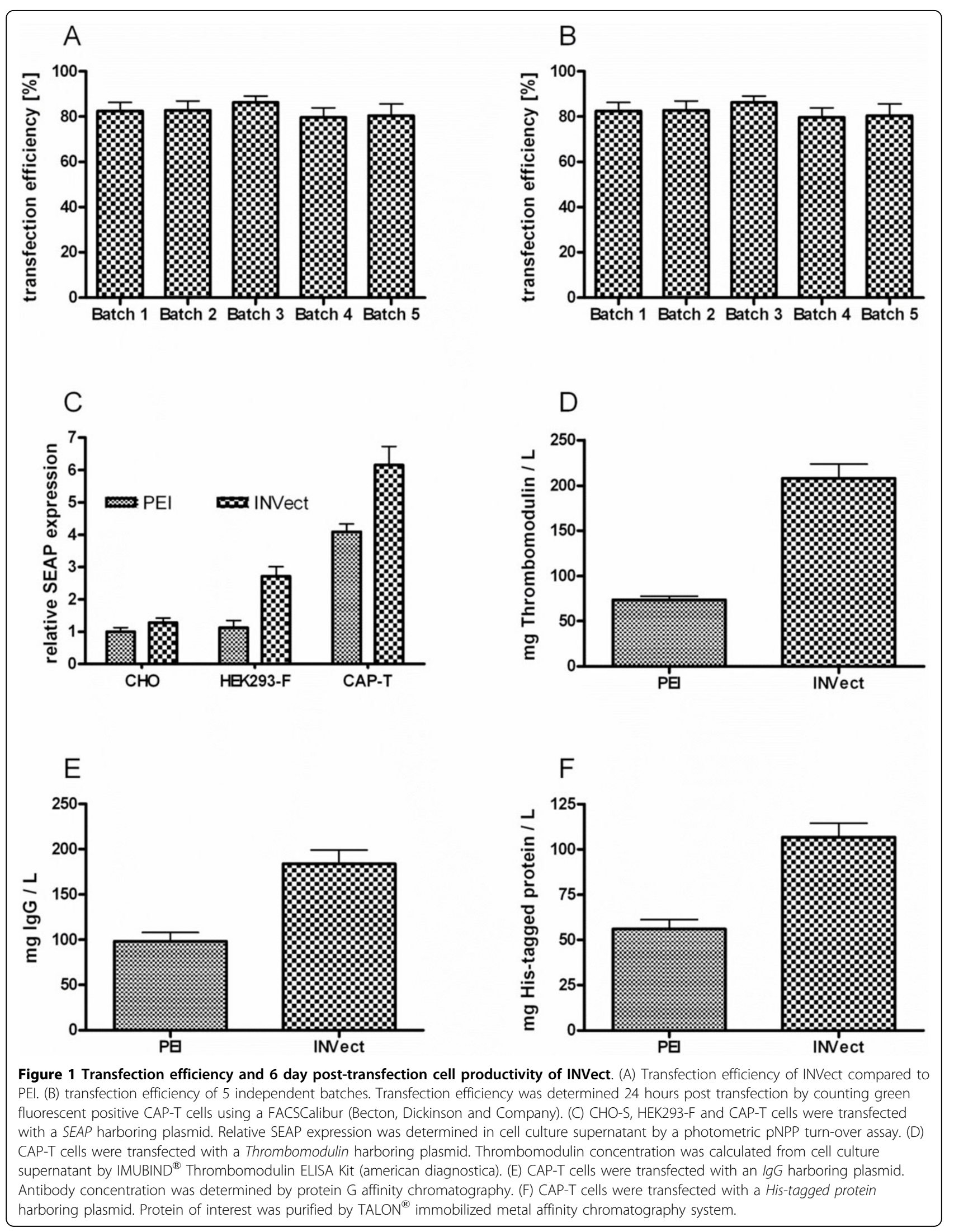


INVect provided a minimum 2-fold increase in protein production over PEI $(25 \mathrm{kDa})$ based transfection.

\section{Conclusions}

INVect is a novel polycationic transfection reagent which demonstrates low cell toxicity for transient transfection of mammalian cells and delivers extremely high transfection efficiencies of up to $90 \%, 24$ h post transfection. The use of INVect for transfection under TGE conditions leads to exceptionally high levels of protein expression and outperforms $25 \mathrm{kDa}$ linear PEI by 2 -fold. INVect can be used effectively with all common cell lines and is especially suited for HEK293-F and CAP-T cells.

\section{Authors' details}

'InVivo BioTech Services GmbH, 16761 Hennigsdorf, Germany. ²emp Biotech $\mathrm{GmbH}, 13125$ Berlin, Germany.

Published: 4 December 2013

\section{References}

1. Geisse S: Reflections on more than 10 years of TGE approaches. Protein Expr Purif 2009, 64:99-107.

2. Fischer S, Charara N, Gerber A, Wölfel J, Schiedner G, Voedisch B, Geisse S: Transient recombinant protein expression in a human amniocyte cell line: the CAP-T ${ }^{\oplus}$ cell system. Biotechnol Bioeng 2012, 109:2250-2261.

doi:10.1186/1753-6561-7-S6-P26

Cite this article as: Püngel et al:: INVect - a novel polycationic reagent

for transient transfection of mammalian cells. BMC Proceedings 20137

(Suppl 6):P26.
Submit your next manuscript to BioMed Central and take full advantage of:

- Convenient online submission

- Thorough peer review

- No space constraints or color figure charges

- Immediate publication on acceptance

- Inclusion in PubMed, CAS, Scopus and Google Scholar

- Research which is freely available for redistribution

Submit your manuscript at www.biomedcentral.com/submit
C Biomed Central 